\title{
Ensino remoto emergencial na percepção de saúde de acadêmicos de Farmácia
}

\author{
Emergency remote education on the health perceptions of Pharmacy academics \\ Enseñanza remota de emergencia en la percepción de salud de académicos de Farmacia
}

Recebido: 25/10/2021 | Revisado: 02/11/2021 | Aceito: 11/11/2021 | Publicado: 17/11/2021

\author{
Ana Paula Lucas Mota \\ ORCID: https://orcid.org/0000-0002-7739-6440 \\ Universidade Federal de Minas Gerais, Brasil \\ Email: analucasmota@gmail.com \\ Gabriel Moreira de Mello Mendes \\ ORCID: https://orcid.org/0000-0003-1427-8401 \\ Universidade Federal de Minas Gerais, Brasil \\ E-mail: gabrielmmmendes2000@gmail.com \\ Rafael Christian de Matos \\ ORCID: https://orcid.org/0000-0003-2644-7305 \\ Universidade Federal de Minas Gerais, Brasil \\ E-mail: rafaelchristiandm@gmail.com \\ Maria do Carmo Vilas Boas Sousa \\ ORCID: https://orcid.org/0000-0001-8779-8323 \\ Universidade Federal de Minas Gerais, Brasil \\ E-mail: mariavilassboas@gmail.com \\ Maria Aparecida Gomes \\ ORCID: https://orcid.org/0000-0002-7263-5721 \\ Universidade Federal de Minas Gerais, Brasil \\ E-mail: magomes@icb.ufmg.br \\ Edmilson Antônio Pereira Júnior \\ ORCID: https:// orcid.org/0000-0003-2837-4744 \\ Universidade Federal de Minas Gerais, Brasil \\ E-mail: pereirajr.edmilson@gmail.com \\ Cristina Mariano Ruas \\ ORCID: https://orcid.org/0000-0003-0275-8416 \\ Universidade Federal de Minas Gerais, Brasil \\ E-mail: crisruasufmg@gmail.com
}

\begin{abstract}
Resumo
Em março de 2020, a Organização Mundial da Saúde caracterizou como pandemia a situação sanitária imposta pelo SARS-CoV-2. Para conter o avanço das infecções, a principal medida governamental foi o distanciamento social. As aulas presenciais foram suspensas e o Ensino Remoto Emergencial (ERE) foi adotado visando minimizar os impactos da pandemia na formação discente. As consequências da pandemia atingiram a vida de todos em diferentes aspectos e, especialmente, na saúde. Neste contexto, avaliaram-se os fatores relacionados à autopercepção de saúde dos discentes do curso de Farmácia da Universidade Federal de Minas Gerais, diante do ERE. Foi aplicado um questionário virtual abordando questões relacionadas ao perfil sociodemográfico, hábitos de vida, aspectos de saúde, prática de atividades físicas e uso de medicamentos. Para análise dos dados, foi utilizado um modelo de árvore de decisão. Um total de 401 discentes respondeu ao questionário. As condições mais importantes relacionadas ao ERE foram não ter tempo para lazer e esportes e sentir-se muito ansioso, exausto e sobrecarregado. A depressão foi a variável com maior discriminação na escala de saúde autopercebida. Para além das incertezas que a pandemia nos traz, o ERE impõe uma nova metodologia de ensino-aprendizagem que preconiza o protagonismo discente para a sua consolidação plena, acentuando sensação de estresse, esgotamento, ansiedade e depressão, caracterizando o adoecimento mental que os alunos estão experimentando. Estes resultados ressaltam a importância do acompanhamento na adaptação ao modelo virtual de ensino, visando seu melhor desenvolvimento e a saúde física e emocional dos discentes.
\end{abstract}

Palavras-chave: Saúde dos estudantes; Pandemia; Ensino superior.

\begin{abstract}
In March 2020, the World Health Organization declared the new coronavirus as pandemic. To contain the infection progressions, the main government through social isolation. At the Federal University of Minas Gerais (UFMG), Emergency Remote Education (ERE) was adopted in order to minimize the impact of the pandemic during the students' education period. The pandemic's consequences have also reached and affected the lives of people around the globe in different social, cultural, economic and, particularly, health aspects. With that in mind, was evaluated the general health conditions of UFMG's Pharmacy's students, before the ERE. A questionnaire was applied addressing issues related to the socio-demographic profile and students' habits, general health, physical activity level, medication
\end{abstract}


use and rate of satisfaction with ERE. A total of 401 students answered the questionnaire (39\% of the total enrolled students). The CART technique was used in data analysis. Stress, tiredness, exhaustion and anxiety were frequent answers in the questionnaire. However, the attribute with the highest discrimination power was the perception of depression. These results are consistent with the atypical moment around the world. Beyond the uncertainties brought upon us by the pandemic, the ERE brings a new teaching-learning methodology that calls for the students' protagonism for its full consolidation, accentuating the feeling of stress, exhaustion, anxiety and depression, characterizing the mental illness that students are experiencing. These results highlight the importance of monitoring in adapting to the virtual teaching model, aiming at their better development and the physical and emotional health of the students.

Keywords: Student health; Pandemic; Education higher.

\section{Resumen}

En marzo de 2020, la Organización Mundial de la Salud declaró la diseminación del nuevo coronavirus una pandemia. Para contener la infección, la principal medida gubernamental fue el distanciamiento social. Se suspendieron las clases presenciales y se adoptó la Enseñanza Remota de Emergencia (ERE) para minimizar los impactos en la educación. Las consecuencias de la pandemia afectaron la vida de todas las personas en diferentes aspectos, como en la salud. En este contexto, se evaluaron los factores relacionados con la autopercepción de la salud de los estudiantes del grado de Farmacia de la Universidad Federal de Minas Gerais, frente al ERE. Se aplicó un cuestionario, con 401 encuestados, abordando el perfil sociodemográfico, estilo de vida, aspectos de salud, actividad física y uso de medicamentos. Para el análisis de datos se utilizó un modelo de árbol de decisión. Las condiciones más importantes relacionadas con ERE fueron no tener tiempo para el ocio y para los deportes y sentirse muy ansioso, exhausto y abrumado. La depresión fue la variable con mayor discriminación en la escala de salud. Además de las incertidumbres que nos trae la pandemia, el ERE impone una nueva metodología de enseñanza-aprendizaje que aboga por el protagonismo estudiantil para su plena consolidación, acentuando los sentimientos de estrés, agotamiento, ansiedad y depresión, caracterizando la enfermedad mental que están viviendo los estudiantes. Estos resultados resaltan la importancia del seguimiento en la adaptación al modelo, con el objetivo de su mejor desarrollo y la salud física y emocional de los estudiantes.

Palabras clave: Salud de los estudiantes, Pandemia, Educación superior.

\section{Introdução}

A situação de pandemia imposta pelo novo Coronavírus (SARS-CoV-2) trouxe múltiplos desafios à sociedade, em diversos setores, públicos e privados, incluindo a saúde e a educação (Sandars \& Patel, 2020). A partir de março de 2020, com a necessidade de isolamento físico e distanciamento social, as práticas cotidianas deram lugar ao trabalho remoto, o que impactou no ensino dentro do contexto das universidades brasileiras (Gusso et al., 2020).

Em Belo Horizonte, Minas Gerais, por meio de decreto municipal, as atividades foram suspensas para enfrentamento da situação de emergência pública em função da pandemia (Belo Horizonte, 2020). No âmbito da UFMG, as atividades presenciais foram suspensas a partir de 18 de março de 2020 (UFMG, 2020). O ensino remoto emergencial (ERE) foi adotado a partir de agosto de 2020, entendendo que este seria o modelo mais adequado para adaptação ao momento sanitário. Questões relacionadas ao processo de ensino-aprendizagem foram avaliadas com o objetivo de conciliar a responsabilidade social da participação dos alunos na atenção à saúde com as demandas de biossegurança impostas pela pandemia (Alvim et al., 2020). Os reflexos deste sistema educacional adaptativo são percebidos em virtude de diferenças socioeconômicas exacerbadas, dificuldades de aprendizado e condições de saúde, evidenciando a necessidade de acompanhamento e avaliação permanente do ERE (Dias \& Pinto, 2020).

Quando se aborda sobre a percepção de saúde, no entanto, tem-se a necessidade de analisar com cautela o grupo amostral escolhido, uma vez que esta percepção perpassa por diferentes vieses. Sob a ótica das variáveis sociodemográficas, por exemplo, tem-se que o gênero é determinante na percepção, uma vez que culturalmente os diferentes gêneros crescem e formam sua consciência com exposições diversas a situações insalubres em ambientes escolares, de trabalho e em momentos de lazer (Barata, 2009). 
De forma análoga, quando o olhar pauta-se nas questões raciais, as condições de crescimento e vivência são oscilantes dentro da diversidade, devido a processos sociais, culturais, históricos e econômicos do país. Desta forma, a percepção de saúde e bem estar se torna modelada segundo estes fatores (Tavares, Oliveira, \& Lages, 2013).

Estudo realizado por Ribeiro et. al., (2016) retrata que alunos ingressantes nas universidades brasileiras através de cotas, oriundos de políticas de ações afirmativas, apresentam rendimento inferior aos não cotistas em cursos mais relacionados às ciências exatas. Essa diferença pode ser ocasionada devido a situações de desvantagem, proveniente de uma possível deficiência do ensino básico (Ribeiro et. al., 2016). Esse problema parece ser um fator estressor, tendo em vista que as situações de desvantagem podem trazer dificuldades na continuação dos estudos, em nível superior e, consequentemente, afetar a saúde do estudante.

Com relação à aprendizagem, as condições de saúde podem interferir na capacidade de foco e atenção dos alunos, na velocidade de processamento da informação e na quantidade e qualidade do conteúdo apreendido. Corroborando com o exposto, a prática de exercício físico relaciona-se com o funcionamento do sistema nervoso central, fazendo com que sua prática, além de proporcionar maior sensação de bem-estar, promova melhoria na capacidade de memorização e de concentração no momento de estudo (Maia et al., 2014).

Ao observar os alunos nota-se, ainda, que estes passam por períodos transformadores e determinantes durante seu trilhar acadêmico. Ao longo dos anos da graduação diversos momentos de pressão ocorrem, gerando sentimentos como ansiedade, estresse e outros distúrbios. O uso de medicamentos é uma alternativa no manejo de doenças diagnosticadas, que pode trazer benefícios clínicos, mas também pode comprometer o aprendizado (Monteiro, Freitas, \& Ribeiro, 2007).

Desde a adoção do modelo remoto no ensino superior, os estudantes estão expostos a situações adversas que transcendem as barreiras cognitivas e tangenciam as questões sociais. A adaptação ao ERE relaciona-se a fatores familiares, sociais e profissionais, que dependem concomitantemente da adaptação universitária diante do contexto de pandemia, para que seja possível a realização das atividades de ensino e a manutenção do estado de saúde da forma menos prejudicial possível (Fior \& Martins, 2020).

Diante deste contexto, o presente trabalho avaliou as condições gerais de saúde de discentes do curso de Farmácia da UFMG, diante do ERE. Os objetivos centrais do estudo foram identificar os aspectos socioeconômicos e de saúde dos discentes do curso de Farmácia da UFMG que se mostraram mais fortemente associados à Escala Geral de Saúde.

\section{Metodologia}

Trata-se de um estudo transversal, realizado para avaliar a autopercepção de saúde dos estudantes do curso de Farmácia da UFMG, por meio de um questionário disponibilizado pela plataforma virtual Google Forms. O estudo insere-se no Programa para o desenvolvimento do ensino de graduação da UFMG (PDEG).

O período de coleta dos dados foi entre 20 e 2809/2020. Um total de 401 discentes respondeu ao questionário (39\% do total de alunos matriculados). O questionário abordou questões relacionadas ao perfil sociodemográfico e hábitos de vida dos discentes. Além disso, foram avaliados aspectos gerais de saúde, prática de atividades físicas e uso de medicamentos.

A variável dependente do estudo foi a "Escala geral de Saúde", avaliada de 0 a 100, onde 0 correspondia ao "pior estado de saúde imaginável" e 100 ao "melhor estado de saúde imaginável".

As variáveis independentes incluíram "sexo", "raça/cor", "período do curso" $\left(1^{\circ}\right.$ ao $13^{\circ}$, dependendo da matriz curricular), "ensino médio em escola pública", "cotas", "principal fonte de renda", "amparado por assistência estudantil", "contemplado com recurso para inclusão digital", "realiza atividade remunerada", "turno", "deficiência", "utilização de medicamentos para o sistema nervoso" e "realização de atividades físicas". 
A avaliação dos aspectos de saúde ocorreu por meio de escala Likert, variando entre 1 e 5 , sendo 1 "discordo totalmente" e 5 "concordo totalmente" e as questões norteadoras foram "me sinto doente por causa do ERE", "me sinto sobrecarregado por causa do ERE", "não tenho tempo para lazer por causa do ERE", "estou pensando em trancar o semestre do ERE", "me sinto exausto", "estou pensando em trancar algumas disciplinas", "me sinto ansioso", "me sinto muito deprimido" e "me sinto doente por causa da pandemia".

Os dados foram analisados por meio da técnica estatística denominada CART (Classification and Regression Tree), utilizada para identificar os fatores que mais discriminaram a variável resposta, ou seja, a Escala Geral de Saúde. Trata-se de uma árvore de decisão construída com base em uma variável resposta e outras 22 variáveis explicativas. A árvore analisada neste estudo é de regressão, pois possui variável resposta quantitativa.

A técnica CART apresenta como principal vantagem um fácil entendimento dos resultados, visto que é possível compreender a estrutura da classificação executada e exibir as subdivisões obtidas. Essa metodologia segue a lógica da construção de árvores através da subdivisão de grupos em subgrupos, sucessivamente (Breisman, 1998). Essas divisões sucessivas seguem o método CHIAD (Chi-square Automatic Interaction Detection), no qual a variável independente é escolhida através da maior interação com as outras variáveis.

Os critérios que foram respeitados no desenvolvimento da técnica CART são: os nós, que se referem aos subconjuntos resultantes da aplicação de uma regra de divisão, devem possuir no mínimo 100 observações para que essa subdivisão seja validada; além disso, cada nó terminal necessita que seja obtido um mínimo de 50 observações e, por fim, foram desconsideradas subdivisões em que a probabilidade estatística (valor de "p") apresentasse valores iguais ou superiores a 0,05 .

O estudo faz parte do projeto intitulado "Acompanhamento de discentes, docentes e egressos da Universidade Federal de Minas Gerais (UFMG)", aprovado pelo Comitê de Ética em Pesquisa da UFMG sob n 15414619.0.0000.5149. O Termo de Consentimento Livre e Esclarecido (TCLE) foi disponibilizado antes da coleta das informações e foi encaminhado automaticamente para o e-mail do aluno respondente.

\section{Resultados e discussão}

Em tempos de crise, as desigualdades sociais e de acesso à tecnologia se acentuam. Sob o ponto de vista da educação, estas diferenças podem ocasionar problemas de saúde que irão gerar consequências no processo de ensino e aprendizado. Neste contexto, avaliamos as condições gerais de saúde dos discentes do curso de Farmácia da UFMG que vivenciaram o ERE.

\section{Perfil dos estudantes respondentes}

O questionário foi aplicado ao longo do primeiro semestre letivo de 2020. Responderam ao questionário 401 alunos, representando 39\% dos matriculados no curso de Farmácia. As características mais prevalentes foram: sexo feminino $(75,1 \%)$, raça/cor branca $(52,1 \%)$, provenientes de escola pública $(55,6 \%)$, cursando do $5^{\circ}$ ao $13^{\circ}$ período $(53,2 \%)$, período diurno $(62,1 \%)$, utilizou sistema de cotas para o ingresso na universidade $(57,9 \%)$ e tinham pais e familiares como principal fonte de renda $(73,3 \%)$. Além disso, 2,5\% dos alunos apresentaram algum tipo de deficiência física e 12,9\% receberam benefícios provenientes da inclusão digital proposta pela UFMG (Tabela 1). 
Tabela 1- Características sociodemográficas dos alunos respondentes do curso de Farmácia-UFMG durante o Ensino Remoto Emergencial, 2020.

\begin{tabular}{|c|c|c|c|}
\hline \multicolumn{2}{|c|}{ Características } & \multicolumn{2}{|l|}{$\mathrm{N}$} \\
\hline \multirow[t]{2}{*}{ Sexo } & Feminino & 301 & 75,1 \\
\hline & Masculino & 100 & 24,9 \\
\hline \multirow[t]{5}{*}{ Cor/raça } & Amarelo & 7 & 1,7 \\
\hline & Branca & 209 & 52,1 \\
\hline & Parda & 136 & 33,9 \\
\hline & Preta & 35 & 8,7 \\
\hline & Não respondeu & 14 & 3,5 \\
\hline \multirow[t]{2}{*}{ Ensino médio em escola pública } & Não & 178 & 44,4 \\
\hline & Sim & 223 & 55,6 \\
\hline \multirow[t]{2}{*}{ Assistido pela Fump } & Não & 275 & 68,6 \\
\hline & Sim & 126 & 31,4 \\
\hline \multirow[t]{2}{*}{ Deficiência } & Não & 391 & 97,5 \\
\hline & Sim & 10 & 2,5 \\
\hline \multirow[t]{3}{*}{ Período } & $1^{\circ}$ ao $4^{\circ}$ & 186 & 46,4 \\
\hline & Do $5^{\circ}$ ao $13^{\circ}$ & 212 & 52,9 \\
\hline & Preferiu não informar & 3 & 0,7 \\
\hline \multirow[t]{2}{*}{ Turno } & Diurno & 249 & 62,1 \\
\hline & Noturno & 152 & 37.9 \\
\hline \multirow[t]{2}{*}{ Cotas } & Não & 232 & 57,9 \\
\hline & Sim & 169 & 42,1 \\
\hline \multirow[t]{4}{*}{$\begin{array}{l}\text { Origem da principal fonte de renda para } \\
\text { se manter como estudante }\end{array}$} & $\begin{array}{l}\text { Bolsas de estágio, iniciação científica, } \\
\text { extensão ou outros }\end{array}$ & 29 & 7,2 \\
\hline & Fundo de assistência estudantil & 32 & 8,0 \\
\hline & Trabalho/aposentadoria & 46 & 11,5 \\
\hline & Pais, marido e/ou familiares & 294 & 73,3 \\
\hline
\end{tabular}




\begin{tabular}{|c|c|c|c|}
\hline \multirow{3}{*}{$\begin{array}{l}\text { Contemplado com recurso para inclusão } \\
\text { digital? }\end{array}$} & Não & 348 & 86,8 \\
\hline & Sim & 52 & 12,9 \\
\hline & Preferiu não informar & 1 & 0,1 \\
\hline Total de alunos & & 401 & 100,0 \\
\hline
\end{tabular}

${ }^{1}$ Fump: Fundação Universitária Mendes Pimentel. Fonte: Elaborado pelos autores (2020).

\section{Condição geral de saúde dos estudantes respondentes}

A autopercepção de saúde dos discentes do curso de Farmácia da UFMG teve média de 69,44+20,09, com variação entre 5 e 100 (Gráfico 1).

Gráfico 1 - Autopercepção de saúde entre discentes do curso de Farmácia-UFMG durante o Ensino Remoto Emergencial, 2020 mensurada por meio de escala que varia entre 0 (pior estado de saúde) e 100 (melhor estado de saúde).

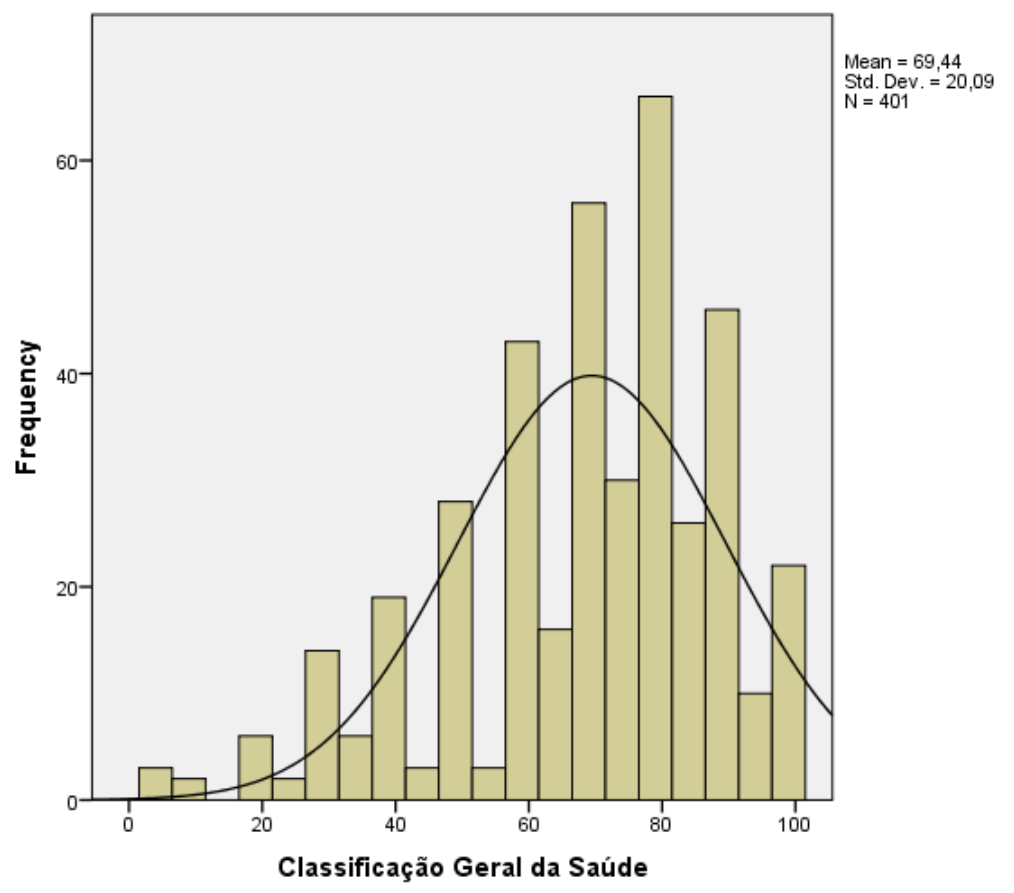

Fonte: Elaborado pelos autores.

As condições de saúde também foram avaliadas por meio de uma escala de concordância com afirmativas que abordavam questões físicas e emocionais. Nesta avaliação, um maior percentual dos estudantes respondeu que não concorda, nem discorda com a afirmativa "me sinto doente por causa da pandemia" (28,2\%); e discorda totalmente com a afirmativa "me sinto doente por causa do ERE” (26,2\%) (Tabela 02). Ressalta-se que, no momento da entrevista a situação epidemiológica apresentava-se estável, em virtude do isolamento social, justificando a sensação de saúde relatada pelos alunos. Os indicadores de monitoramento, ao final do mês de Outubro de 2020, estavam em níveis verdes, ou seja, dentro dos limites aceitáveis, sendo o número médio de transmissão por infectado igual a 0,89; ocupação dos leitos de UTI Covid igual a 30,2\% e ocupação dos leitos de enfermaria Covid igual a 28,2\% (SMS-PBH, 134/2020). 
Os dados que mais chamam a atenção estão relacionados às maiores proporções de concordância total com as seguintes afirmativas: "não tenho tempo para lazer e esportes por causa do ERE" (32,4\%); "eu me sinto muito ansioso" (47,4\%); "eu me sinto exausto" (55,4\%) e "eu me sinto sobrecarregado com o ERE" (50,6\%) (Tabela 02). Neste contexto, devemos salientar que o ERE trouxe inúmeros desafios para os discentes, dentre eles o grande tempo dispensado em frente à tela do computador, o gerenciamento do tempo para o aprendizado e outras atividades e o domínio das tecnologias digitais. As demandas estudantis se misturaram às demandas familiares. A inabilidade na gestão dessas atividades pode ter culminado com a ansiedade e a exaustão, referidas pelos alunos.

Proporções similares dos diferentes níveis de concordância foram observadas na afirmativa "eu me sinto muito deprimido" (Tabela 2). Este dado será detalhado adiante, a partir da apresentação dos resultados utilizando-se a CART.

Tabela 02: Distribuição da percepção da saúde física e emocional dos alunos de graduação de Farmácia-UFMG durante o Ensino Remoto Emergencial (ERE), 2020.

\begin{tabular}{l|l|l|l|l|l}
\hline & $\begin{array}{l}\text { (Discordo } \\
\text { totalmente) }\end{array}$ & 2 & 3 & 4 & $\begin{array}{l}5 \text { (Concordo } \\
\text { totalmente) }\end{array}$ \\
\hline $\begin{array}{l}\text { Me sinto doente por causa da } \\
\text { pandemia }\end{array}$ & $25,2 \%$ & $20,2 \%$ & $28,2 \%$ & $16,2 \%$ & $10,2 \%$ \\
\hline $\begin{array}{l}\text { Me sinto doente por causa do } \\
\text { ERE }\end{array}$ & $26,2 \%$ & $22,4 \%$ & $20,7 \%$ & $17,0 \%$ & $13,7 \%$ \\
\hline $\begin{array}{l}\text { Eu não tenho tempo para lazer } \\
\text { e esportes por causa do ERE }\end{array}$ & $15,5 \%$ & $13,5 \%$ & $20,2 \%$ & $18,5 \%$ & $32,4 \%$ \\
\hline Eu me sinto muito ansioso & $6,2 \%$ & $9,0 \%$ & $11,7 \%$ & $25,7 \%$ & $47,4 \%$ \\
\hline Eu me sinto muito deprimido & $20,2 \%$ & $20,4 \%$ & $20,7 \%$ & $18,0 \%$ & $20,7 \%$ \\
\hline Eu me sinto exausto & $4,5 \%$ & $6,7 \%$ & $14,7 \%$ & $18,7 \%$ & $55,4 \%$ \\
\hline $\begin{array}{l}\text { Eu me sinto sobrecarregado } \\
\text { com o ERE }\end{array}$ & $7,2 \%$ & $6,2 \%$ & $18,0 \%$ & $18,0 \%$ & $50,6 \%$ \\
\hline
\end{tabular}

Fonte: Elaborada pelos autores (2020).

A saúde autopercebida baseia-se em critérios subjetivos e objetivos e parte de uma construção individual, com influência dos fatores biológicos, psicológicos e sociais (Jylhâ et al., 19980. É essencial entender como a pessoa percebe sua saúde, sobretudo estudantes, pois todo o processo de ensino-aprendizado permeia a saúde. Assim, diversos fatores, chamados determinantes sociais da saúde, vão influenciar nesta percepção. Em estudantes, questões econômicas, culturais, políticas, familiares e relacionadas ao acesso explicam, pelo menos em parte, os resultados encontrados.

O ensino pode representar elevada carga no adoecimento dos estudantes. Estudos realizados em diferentes países têm mostrado o impacto da pandemia na saúde mental dos indivíduos, com aumento dos casos de depressão e ansiedade (De Boni et al. 2020; Wang et. al, 2020). Assim, desde o início, a Pró-reitoria de graduação da UFMG (Prograd) definiu orientações para a implantação do ERE de forma a mitigar seus efeitos estressores nos alunos. As orientações incluíram ações como: manutenção das aulas nos mesmos dias e turnos do ensino presencial, visando preservar a rotina dos estudantes e garantir o 
horário dos encontros síncronos; o tempo de aulas não poderia ultrapassar a carga horária de cada disciplina e, ainda, computar o tempo de leitura e de realização de exercícios na carga horária da disciplina. Coube aos alunos o gerenciamento do tempo e a seleção das disciplinas cursadas. O formato virtual das atividades e o tempo gasto para os estudos podem ter levado à ansiedade e exaustão, seja pelo excesso de conteúdo ou pela inabilidade de gerir o tempo de acordo com a autonomia imposta pelo ensino remoto. Somam-se aos pontos apresentados questões individuais no aprendizado, o tempo de adaptação à nova rotina e à nova tecnologia de ensino e aprendizado, além dos contratempos do ambiente virtual. Ressalta-se que a situação de isolamento físico por si só pode vir a ocasionar situações de ansiedade e depressão, tendo em vista a característica social do ser humano (Bittencourt, 2020).

Outras questões, relacionadas ao momento vivenciado, tais como perdas econômicas e familiares, medos e incertezas, também influenciam, direta ou indiretamente, na saúde dos indivíduos (Brooks et al., 2020; Gomes \& Favorito, 2020).

Embora não se tenha realizado uma análise do perfil econômico dos alunos, variáveis como a origem (escola pública ou privada) e utilização de recursos financeiros de instituições de apoio estudantil podem ser utilizadas como uma proxi das condições dos alunos. Considerando-se que mais da metade dos estudantes são provenientes de escola pública e mais de um terço utiliza recursos da Fump (Tabela 01), infere-se que a maioria dos alunos possui baixas condições econômicas. Isto vai se traduzir em elevado número de pessoas e níveis de ruídos no domicílio, que podem perturbar a concentração nos estudos; espaço físico limitado; baixa disponibilidade de alimentação e necessidade de trabalho para sustento. Estas questões podem interferir na saúde dos estudantes, seja por competirem com o tempo de estudo, seja dificultando o seu acesso.

De acordo com Paula, (2017), o cenário de desigualdades raciais, econômicas e de acesso tecnológico já era preocupante antes da pandemia, uma vez que, embora existissem políticas de inclusão social, as políticas de permanência no ensino superior eram insuficientes. Assim, com a pandemia, as preocupações com estudantes de baixas condições socioeconômicas se exacerbaram. Nesse sentido, várias universidades implantaram políticas de inclusão digital, viabilizando auxílio financeiro para aquisição de equipamentos, pacotes de dados de internet, visando democratizar o acesso tecnológico, essencial ao ERE (Melo et al., 2020). A UFMG, via Prograd (2020), abriu edital de auxílio à inclusão digital. Ainda assim, outras dificuldades de acesso à internet, traduzidas como falhas de conexão e problemas nos equipamentos, podem ter contribuído para um aumento de estresse nos alunos.

Paralelamente, estudantes com deficiências já apresentam necessidades diferenciadas em um contexto sanitário normal. Com a pandemia, as dificuldades encontradas no ERE podem desencadear fatores estressores para a saúde. Como exemplo dessas condições de deficiências, podemos citar alunos com deficiência visual, os quais já sofrem dificuldades relacionadas à acessibilidade e permanência no formato presencial. Estes necessitam de maior auxílio para a realização das atividades no modelo remoto, tendo em vista a grande quantidade de atividades relacionadas à leitura, que devem ser adaptadas ao discente que apresenta essa deficiência (Leite, Silva, Simões, Silva, \& Pereira, 2020). Além desses, existem os alunos com baixa acuidade auditiva, que realizam leituras labiais durante o ensino presencial. No formato virtual, nem sempre as legendas aparecem e, muitas vezes, o professor não aparece na vídeo-aula gravada (De Oliveira Silva, De Souza Santos, \& Cury et al., 2020; Vasconcelos, 2020).

Questões relacionadas à sobrecarga das mulheres são recorrentes na sociedade brasileira e influenciam diretamente na saúde desta população. Como, neste estudo, representam 75,1\% dos respondentes, compreende-se o perfil de adoecimento encontrado. Estudo realizado na mesma instituição em 2018 encontrou que os fatores mais fortemente relacionados a uma má percepção de saúde foram sexo feminino e o fato de possuírem vínculo empregatício (Ruas \& Pereira Junior, 2021). O fato de estudar, trabalhar e ainda ter que arcar com os serviços domésticos, muitas vezes sozinhas, são fatores que podem influenciar negativamente na saúde. 
Detectou-se que, somente, 36,4\% dos alunos praticam alguma atividade física. Estudos apresentam a prática de exercícios físicos, através de atividades como caminhar, correr ou pedalar, como fundamental para manter a saúde (Andrade, 2011; Maia et al., 2014). O sedentarismo leva a doenças como hipertensão, diabetes, obesidade e distúrbios cardiovasculares, que contabilizam cerca de dois milhões de mortes anualmente (Mandsager et al. 2018). Além disso, o cognitivo também é beneficiado com a prática dessas atividades (Antunes et al., 2006). Somando a esses pontos, a qualidade de vida e o bem estar de universitários é, também, fortemente influenciado por atividade física e alimentação adequada (Marcondeli, Costa, \& Schmitz, 2008; Fontes, \& Vianna, 2009; Maia et. al, 2014). Neste momento excepcional que vivenciamos, a atividade física poderia constituir uma válvula de escape para amenizar os sintomas de ansiedade e depressão, melhorando a saúde dos estudantes e, por consequência, seu rendimento escolar.

Dos entrevistados, 22,2\% utilizavam algum medicamento para o sistema nervoso, sendo os mais prevalentes os ansiolíticos (24\%) e antidepressivos (17\%). O estresse, o cansaço, a depressão e ansiedade apresentados pela maioria dos alunos entrevistados é consistente com o momento vivido na pandemia. Além disso, o ERE trouxe uma metodologia de ensinoaprendizado adaptada ao meio virtual, impondo o protagonismo do aluno para sua consolidação. Neste formato de ensino a autonomia dos estudantes é fundamental.

A análise dos fatores socioeconômicos, demográficos e acadêmicos associados à saúde dos discentes foi analisada por meio da técnica estatística CART. O modelo realizou divisões sucessivas da amostra de forma a considerar os atributos com maior poder de discriminação entre os subgrupos. Entre elas, a "percepção da depressão" foi a variável com maior discriminação na classificação da saúde dos alunos, cujas médias da escala foram menores para os maiores níveis de concordância com a auto-percepção de depressão. A partir de subdivisões sucessivas, somente a variável "turno de estudo" foi significativa para diferenciar os estudantes que concordavam parcialmente com este sentimento, sendo que a média da escala de saúde foi menor para os estudantes do diurno $(55,9 \pm 19,7)$ comparando-se ao noturno $(63 \pm 20,3)$ (Figura 1). 
Figura 1 - Árvore de regressão da escala de saúde de acordo com as características socioeconômicas, demográficas e acadêmicas dos discentes do Curso de Farmácia-UFMG durante o Ensino Remoto Emergencial, 2020.

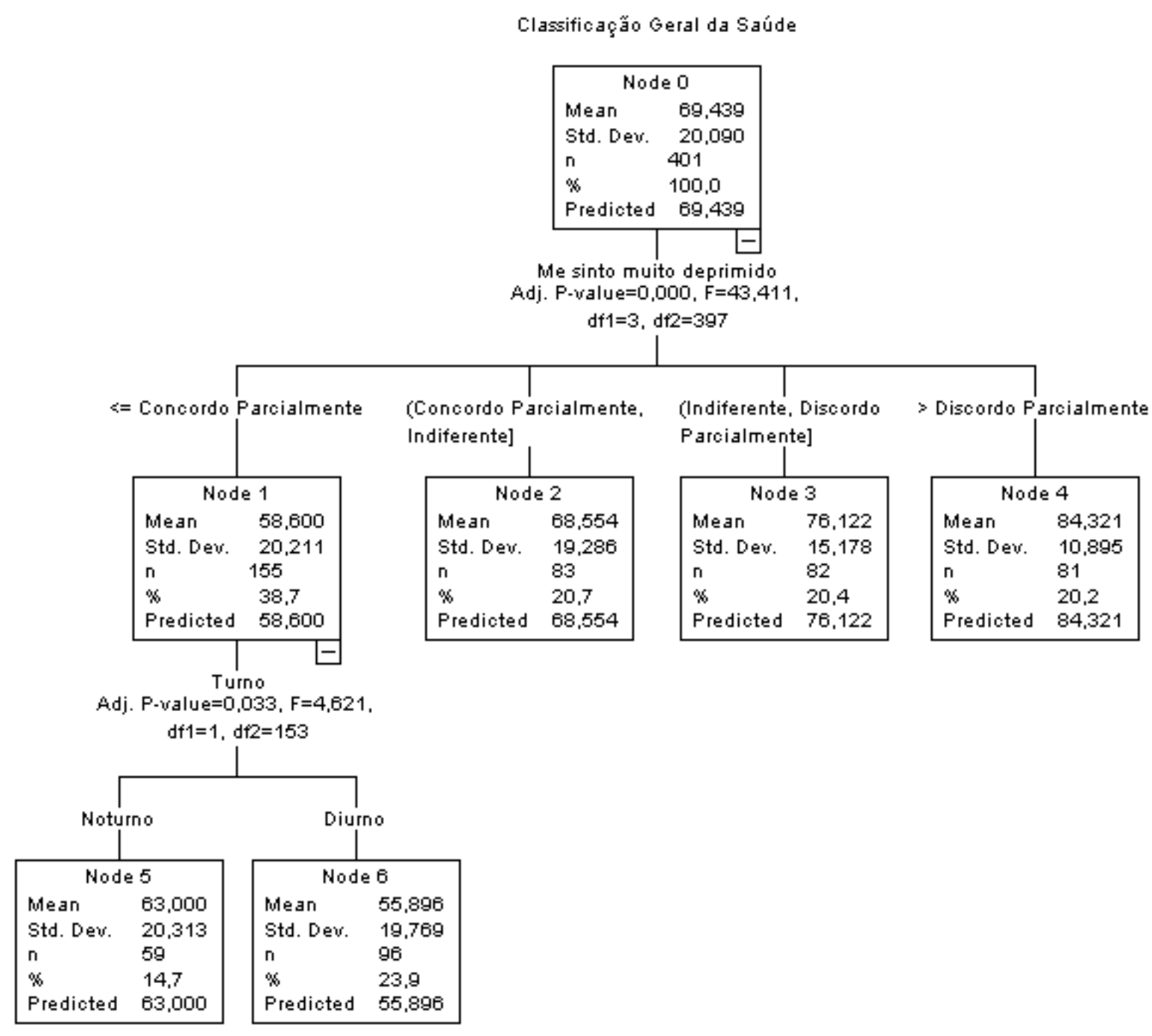

Fonte: Elaborado pelos autores (2020).

A depressão foi o fator de maior influência na percepção de saúde dos alunos. Corrobora com estes dados, um estudo que relatou elevada prevalência de depressão entre alunos de áreas da saúde (27\%) (Ribeiro, Da Cruz, Marchi, Tirapelli, Miasso, 2014). Segundo dados do IBGE (2020), a taxa de depressão diagnosticada por profissionais de saúde entre brasileiros acima de 18 anos é estimada em 10,2\%. Contudo, o modelo de ensino remoto pode ter acentuado os sintomas da depressão, ansiedade e estresse no processo de ensino-aprendizagem, fazendo com que seja necessária atenção à adaptação estudantil para contenção de efeitos danosos (Santos, Silva, \& Belmonte, 2021).

Buscando um acolhimento aos estudantes necessitados, além de reduzir índices de retenção e evasão no curso de farmácia, a Faculdade de Farmácia da UFMG tem um projeto institucional de Escuta Acadêmica, desenvolvido desde 2016, que envolve escuta individual e rodas de conversa coletiva, a partir de demandas do próprio aluno, dos colegas ou do professor e a partir de indicadores institucionais. Resultados da efetividade do projeto ainda não foram publicados. Entretanto, os alunos 
têm relatado inúmeros fatores positivos no acompanhamento estudantil, seja na intermediação da comunicação discentedocente; no encaminhamento aos serviços de saúde ou de assistência estudantil; auxílio na organização e gestão do tempo e práticas de meditação e relaxamento. Assim, ações como estas poderiam ser úteis para auxiliar os discentes durante o período de adaptação estudantil e como suporte nos momentos mais estressantes do curso.

Dentre os alunos que alegaram concordar parcialmente com a afirmativa "Me sinto muito deprimido", o fator de maior associação com a percepção de saúde foi o turno, sendo que os alunos do turno diurno apresentaram percepção de saúde pior que os do turno noturno. Desta forma, infere-se que, provavelmente, a média de saúde no diurno foi menor, pois os cursos diurnos apresentam maior carga horária de aula semestral, fazendo com que os alunos tenham que despender mais energia, atenção e tempo para as atividades, podendo, em caso de má gestão do tempo, gerar sobrecarga superior no modelo remoto.

\section{Considerações Finais}

O ERE é um formato provisório, mas que tem trazido preocupações, não só pela longa duração e imprevisibilidade do seu término, mas também pelas consequências na saúde dos estudantes a longo prazo. As condições mais importantes, descritas neste estudo e relacionadas à saúde, foram falta de tempo para lazer e esportes por causa do ERE e sentimentos de ansiedade, exaustão e sobrecarga com o ERE. Ações voltadas para melhor gerenciamento do tempo e garantia de cumprimento de medidas organizativas do ERE podem ser úteis para mitigar estes efeitos.

A depressão foi a variável com maior discriminação na escala de saúde autopercebida. Além disso, cerca de 1/4 dos estudantes utilizavam algum medicamento para o sistema nervoso, sendo os mais prevalentes os ansiolíticos e antidepressivos. Ressalta-se a importância do acompanhamento na adaptação ao modelo virtual de ensino, visando seu melhor desenvolvimento e a preservação da saúde física e emocional dos discentes. Ademais, acompanhamento longitudinal, ações voltadas para a promoção da saúde dos estudantes será muito importante no retorno ao ensino presencial, tendo em vista o momento vivenciado.

\section{Agradecimentos}

A Pró-reitoria de Graduação (Prograd) - UFMG, por subsidiar o estudo através do Programa para o Desenvolvimento do Ensino de Graduação (PDEG)

\section{Referências}

Alvin, C. G., Medeiros, A. M., Penna, C. F. A. M., Ruas, C. M., Vilaça, E. L., Lourenço, E., Ferreira, F. R., Baroni, F. C. A. L., Mota, L. G., \& Rocha, M. G. L. (2020) Cursos da Saúde. Revista Docência do Ensino Superior, 10, 1-21. https://doi.org/10.35699/2237-5864.2020.24767

Andrade, T.bR. (2011) O exercício físico no tratamento da depressão: uma revisão de literatura. Trabalho de Conclusão de Curso (Faculdade de Educação Física) Universidade Estadual de Campinas. http:// https://www.passeidireto.com/arquivo/70112503/o-exercicio-fisico-no-tratamento-da-depressao

Antunes, H. K. M., Santos, R. F., Cassilhas, R., Santos, R. V. T., Bueno, O. F. A., \& Mello, M. T. (2006) Exercício físico e função cognitiva: uma revisão. Revista Brasileira de Medicina do Esporte, 12(2), 108-114. https://doi.org/10.1590/S1517-86922006000200011

Barata, R. B. (2009). Relações de gênero e saúde: desigualdade ou discriminação? In: Como e por que as desigualdades sociais fazem mal à saúde [online]. Rio de Janeiro: Editora FIOCRUZ. Temas em Saúde collection, pp. 73-94. https://static.scielo.org/scielobooks/48z26/pdf/barata-9788575413913.pdf

BELO HORIZONTE (2020). Prefeitura Municipal. Decreto n ${ }^{\circ}$ 17.304, de 18 de março de 2020. Determina a suspensão temporária dos Alvarás de Localização e Funcionamento e autorizações emitidos para realização de atividades com potencial de aglomeração de pessoas para enfrentamento da Situação de Emergência Pública causada pelo agente Coronavírus - COVID-19. Belo Horizonte. https://www.cmbh.mg.gov.br/atividade-legislativa/pesquisarlegislacao/decreto/17304/2020.

Bittencourt, R. N (2020). Pandemia, isolamento social e colapso global. Revista Espaço Acadêmico, 19 (221), 168-178. https://periodicos.uem.br/ojs/index.php/EspacoAcademico/article/view/52827

De Boni, I. R. B., Balanzá-Martínez, V., Mota, J. C., Cardoso, T. A., Ballester, P., Atienza-Carbonell, B., Bastos, F. I., \& Kapczinski, F. (2020). Depression, Anxiety, and Lifestyle Among Essential Workers: a web survey from Brazil and Spain during the covid-19 pandemic. Journal of Medical Internet Research, 22 (10) 1-16. https://www.arca.fiocruz.br/handle/icict/45005 
Breiman, L., Friedman, J. H., Olshen, R. A., \& Stone, C. J. L. (1998). Classification and Regression Trees. Chapman and Hall 368 Pages.

Brooks, S. K., Webster, R. K., Smith, L. E., Woodland, L., Wessely, S., Greenberg, N., \& Rubin, G. J. (2020). The psychological impact of quarantine and how to reduce it: rapid review of the evidence. The lancet, 395, 912-920. https://doi.org/10.1016/S0140-6736(20)30460-8

De Oliveira Silva, C., De Souza Santos, F., \& Cury, I. L. R (2020). Desafios e possibilidades da inclusão de surdos no ensino superior. Educação, Ensino e Docência, p. 76-86. http://www.foxtablet.com.br/wp-content/uploads/2021/01/Educacao_ensino_docencia.pdf\#page=77

Dias, E., \& Pinto, F. C. F. (2020). A Educação e a Covid-19. Ensaio: Avaliação e Políticas Públicas em Educação, 28 (108), 545-554. https://doi.org/10.1016/S0140-6736(20)30460-8

Filho, C. A. A. M., Alves, C. R. R., Sepúlveda, C. A., Costa, A. S., Lancha Junior, A. H., \& Gualano, B. (2014). Influência do exercício físico na cognição: uma atualização sobre mecanismos fisiológicos. Revista Brasileira Medicina e Esporte, 20 (30 237-241. http://dx.doi.org/10.1590/1517-86922014200301930

Fior, C. A., \& Martins, M. J (2020). A docência universitária no contexto de pandemia e o ingresso no ensino superior. Revista Docência do Ensino Superior, 10, 11-20. https://doi.org/10.35699/2237-5864.2020.24742

Fontes, A. C. D., \& Vianna, R. P. T. (2009) Prevalência e fatores associados ao baixo nível de atividade física entre estudantes universitários de uma universidade pública da região Nordeste - Brasil. Revista Brasileira de Epidemiologia, 12 (1), 20-29. https://doi.org/10.1590/S1415-790X2009000100003

Gomez, A. M. A., \& Favorito, L. A. (2020) The Social, Economic and Sanitary Impact of COVID-19 Pandemic. International Brazilian Journal Urology, 46, 3-5. https://doi.org/10.1590/S1677-5538.IBJU.2020.S1ED2

Gusso, H. L., Archer, A. B., Sahão L. F. B., Luca, G. G., Henklain, M. H. O., Panosso, M. G., Kienen, N., Beltramello, O., \& Gonçalves, V. M. (2020). Ensino superior em tempos de pandemia: diretrizes à gestão universitária. Educação \& Sociedade, 41, 1-27. https://doi.org/10.1590/ES.238957

Heitzman, J. (2020). Impact of COVID-19 pandemic on mental health. Psychiatria Polska, 54 (2), 187-198. 10.12740/PP/120373. Epub 2020 Apr 30

IBGE (2020). Pesquisa nacional de Saúde 2019: Percepção do estado de saúde, estilo de vida, doenças crônicas e saúde bucal - Brasil e grandes regiões. Rio de Janeiro. https://biblioteca.ibge.gov.br/index.php/biblioteca-catalogo?view=detalhes\&id=2101764

Leite, L., Silva, M. C. R., Simões, T. M. S., Silva, A. C. S., \& Pereira, M. (2020). Impactos da COVID-19 na graduação da pessoa com deficiência visual. Revista Encantar: Educação, Cultura e Sociedade, Bom Jesus da Lapa, 2, 1-14. https://www.revistas.uneb.br/index.php/encantar/article/view/8863

Lima, F. C., Costa, S. P., Rodrigues, B. M., Matos, D. G., Venturini, G. R. O., Aidar, F. J., \& Mazini Filho, M. (2014). Treinamento resistido na Educação Física Escolar: uma proposta de ensino. Cinergis, Santa Cruz do Sul, 15 (4), 207-217. https://doi.org/10.17058/cinergis.v15i4.5452

Lipp, M. E. N. (1996). Stress: conceitos básicos. In: Pesquisas sobre stress no Brasil: saúde ocupações e grupos de risco. Campinas: Papirus. p. 17-31.

Maia, D. A. C., Vasconcelos, J. A., Vasconcelos, L. A., \& Filho, J. O. V. (2014) Acadêmicos de medicina e a prática de atividade física. Coleção Pesquisa em Educação Física, Várzea Paulista, 13 (1), 15-22. https://fontouraeditora.com.br/periodico/upload/artigo/1083_1503948701.pdf

Mandsager, K., Harb, S., Cremer, P., Phelan, D., Nissen, S. E., \& Jaber, W. (2018). Association of Cardiorespiratory Fitness With Long-term Mortality Among Adults Undergoing Exercise Treadmill Testing. JAMA Network Open, 1 (6), 183605. 10.1001/jamanetworkopen.2018.3605

Marcondelli, P., Costa, T. H. M., \& Schmitz, B. A. S. (2008). Nível de atividade física e hábitos alimentares de universitários do $3^{\circ}$ aos $5^{\circ}$ semestres da área da saúde. Revista de Nutrição, 21 (1), 39-47. https://doi.org/10.1590/S1415-52732008000100005

Mélo, C. B., Farias, G. D. Moises, L. S., Bezerra, L. R. M., \& Piagge, C. S. L. D. (2020). Ensino remoto nas universidades federais do Brasil: desafios e adaptações da educação durante a pandemia de covid-19. Research, Society and Development, 9 (11), 1-19. https://doi.org/10.33448/rsd-v9i11.9866

Monteiro, C. F. S., Freitas, J. F. M., \& Ribeiro, A. A. P. (2007). Estresse no cotidiano acadêmico: o olhar dos alunos de Enfermagem da Universidade Federal do Piauí. Escola Anna Nery Revista de Enfermagem, 11 (1), 66-72. https://doi.org/10.1590/S1414-81452007000100009

Paula, M. F. C. (2017). Políticas de democratização da educação superior brasileira: limites e desafios para a próxima década. Avaliação: Revista da Avaliação da Educação Superior, 22 (2), 301-315. http://dx.doi.org/10.1590/s1414-40772017000200002.

PROGRAD UFMG (2020). UFMG lança política de inclusão digital para garantir acesso ao ensino remoto emergencial. Belo Horizonte. https://www2.ufmg.br/prograd/prograd/Pro-Reitoria-de-Graduacao/Noticias/UFMG-lanca-politica-de-inclusao-digital-para-garantir-acesso-ao-ensino-remotoemergencial

Ribeiro, A. G., Da Cruz, L. P., Marchi, K. C., Tirapelli, C. R., \& Miasso, A. I. (2014). Antidepressivos: uso, adesão e conhecimento entre estudantes de medicina. Ciência \& Saúde Coletiva, 19 (6), 1825-1833. https://doi.org/10.1590/1413-81232014196.06332013

Ribeiro, S. M., Almeida, M. T. C., Maia, F. A., Souza, F. A., Amaral, D. P. O., \& Alves, M. R. (2016). Política de cotas no ensino superior: Percepções de professores e estudantes. Revista Unimontes Científica. 18 (1), 64-73. https://www.periodicos.unimontes.br/index.php/unicientifica/article/view/1904

Ruas, C. M., \& Pereira Junior, E. A. (2020). Percepções dos discentes do curso de Farmácia de uma instituição de ensino superior. Revista Internacional de Educação Superior, 7, 1-18. https://doi.org/10.20396/riesup.v7i0.8655138

Sandars, J., \& Patel, R. (2020). The challenge of online learning for medical education during the COVID-19 pandemic. International Journal of Medical Education, 11, 169-170. https://doi.org/10.5116/ijme.5f20.55f2

Santos, G. M. R. F., Silva, M. E., \& Belmonte, B. R. (2021). COVID-19: ensino remoto emergencial e saúde mental de docentes universitários. Revista Brasileira de Saúde Materno Infantil, 21 (1), 237-243. https://doi.org/10.1590/1806-9304202100S100013 
Research, Society and Development, v. 10, n. 15, e31101522292, 2021

(CC BY 4.0) | ISSN 2525-3409 | DOI: http://dx.doi.org/10.33448/rsd-v10i15.22292

SMS-PBH - Boletim epidemiológico e assistencial Covid-19 no 134/2020, publicado em 29/10/2020. https://prefeitura.pbh.gov.br/sites/default/files/estruturade-governo/saude/2020/boletim_epidemiologico_assistencial_134_covid-19_29-10-2020.pdf

Tavares, N. O., Oliveira, L. V., \& Lages, S. R. C. (2013). A percepção dos psicólogos sobre o racismo institucional na saúde pública. Saúde debate, 37 (99), 580-587. https://www.scielo.br/j/sdeb/a/jCfZVYPGcL9Sff8MpgtWK6z/?format=pdf\&lang=pt

Universidade Federal De Minas Gerais (2020). PORTARIA N ${ }^{\circ}$ 1819, DE 18 DE MARÇO DE $2020 . \quad$ Belo Horizonte. https://ufmg.br/storage/9/d/5/b/9d5bff9213dc34c2ed1763bb4c4143ea_15845832990431_378828222.pdf

Vasconcelos, I. L. (2020). O desafio da acessibilidade de alunos surdo na perspectiva do ensino remoto. In. Congresso Internacional de Educação Inclusiva, Anais IV CINTED, Campina Grande, Realize Editora.

https://editorarealize.com.br/editora/anais/cintedi/2020/TRABALHO_EV137_MD4_SA7_ID846_25092020150739.pdf

Wang, X., Hegde, S., Son, C., Keller, B., Smith, A., \& Sasangohar, F. (2020). Investigating mental health of US college students during the COVID-19 pandemic: cross-sectional survey study. Journal of Medical Internet Research, 22 (9), e22817. https://preprints.jmir.org/preprint/22817

Jylhâ, M, Guralnik, J. M., Ferrucci, L., Jokela, J., \& Heikkinen, E. (1998). Is self-rated health comparable across cultures and genders? Journal of Gerontology B Psychological Sciences and Social Sciences, 55(3):S144-144-152. 10.1093/geronb/53b.3.s144. 